\title{
Spitzer observations of SN 2014J and properties of mid-IR emission in Type Ia supernovae
}

\author{
J. Johansson, ${ }^{1 \star}$ A. Goobar, ${ }^{1}$ M. M. Kasliwal, ${ }^{2}$ G. Helou, ${ }^{3}$ F. Masci, ${ }^{3}$ S. Tinyanont, ${ }^{4}$ \\ J. Jencson, ${ }^{2}$ Y. Cao, ${ }^{2}$ O. D. Fox, ${ }^{5}$ M. Kromer, ${ }^{6}$ R. Amanullah, ${ }^{1}$ D. P. K. Banerjee, ${ }^{7}$ \\ V. Joshi, ${ }^{7}$ A. Jerkstrand, ${ }^{8}$ E. Kankare ${ }^{8}$ and T. A. Prince ${ }^{2}$ \\ ${ }^{1}$ Department of Physics, The Oskar Klein Centre, Stockholm University, SE 10691 Stockholm, Sweden \\ ${ }^{2}$ Division of Physics, Mathematics and Astronomy, California Institute of Technology, Pasadena, CA 91125, USA \\ ${ }^{3}$ Infrared Processing and Analysis Center, California Institute of Technology, M/S 100-22, Pasadena, CA 91125, USA \\ ${ }^{4}$ Harvey Mudd College, 301 Platt Boulevard, Claremont, CA 91711, USA \\ ${ }^{5}$ Department of Astronomy, University of California, Berkeley, CA 94720-3411, USA \\ ${ }^{6}$ Department of Astronomy, The Oskar Klein Centre, Stockholm University, SE 10691 Stockholm, Sweden \\ ${ }^{7}$ Astronomy and Astrophysics Division, Physical Research Laboratory, Navrangpura, Ahmedabad 380009, India \\ ${ }^{8}$ Astrophysics Research Center, School of Mathematics and Physics, Queen's University Belfast, BT7 1NN, UK
}

Accepted 2016 December 20. Received 2016 December 1; in original form 2016 December 1

\begin{abstract}
SN 2014J in M 82 is the closest Type Ia supernova (SN Ia) in decades. The proximity allows for detailed studies of supernova physics and provides insights into the circumstellar and interstellar environment. In this work, we analyse Spitzer mid-infrared (mid-IR) data of SN 2014J in the 3.6 and $4.5 \mu \mathrm{m}$ wavelength range, together with several other nearby and well-studied SNe Ia. We compile the first composite mid-IR light-curve templates from our sample of SNe Ia, spanning the range from before peak brightness well into the nebular phase. Our observations indicate that $\mathrm{SNe}$ Ia form a very homogeneous class of objects at these wavelengths. Using the low-reddening supernovae for comparison, we constrain possible thermal emission from circumstellar dust around the highly reddened SN 2014J. We also study SNe 2006X and 2007le, where the presence of matter in the circumstellar environment has been suggested. No significant mid-IR excess is detected, allowing us to place upper limits on the amount of pre-existing dust in the circumstellar environment. For SN 2014J, $M_{\text {dust }} \lesssim 10^{-5} \mathrm{M}_{\odot}$ within $r_{\text {dust }} \sim 10^{17} \mathrm{~cm}$, which is insufficient to account for the observed extinction. Similar limits are obtained for SNe 2006X and 2007le.
\end{abstract}

Key words: circumstellar matter-supernovae: general-supernovae: individual: 2005df, 2006X, 2007af, 2007le, 2007sr, 2009ig, 2012cg, 2011fe, 2014J -dust, extinction.

\section{INTRODUCTION}

The use of Type Ia supernovae ( $\mathrm{SNe}$ Ia) as distance indicators remains essential for the study of the expansion history of the Universe and for exploring the nature of dark energy (see e.g. review by Goobar \& Leibundgut 2011). However, a lack of understanding of the progenitor systems and the requirement for empirically derived colour-brightness corrections represent severe challenges for precision cosmology. SN 2014J in the starburst galaxy M 82 is the closest SN Ia in decades and offers a unique opportunity to study progenitor and explosion models, as well as the circumstellar (CS) and interstellar (IS) medium along the line of sight across an unprecedented wavelength range, from gamma-rays to radio (Diehl et al. 2014; Pérez-Torres et al. 2014).
Our Spitzer observations of SN 2014J, along with observations of other well-studied nearby SNe Ia, allow us to compile the first mid-infrared (mid-IR) light-curve templates, presenting us with a new wavelength range to confront theoretical models of SNe Ia. For example, the study of the mid-IR light curve around the nearIR secondary maximum, shown by Kasen (2006) to be a valuable diagnostic of the physical parameters governing SN Ia explosions, could be used to test the validity of the model predictions.

Furthermore, by searching for excess mid-IR emission towards SN 2014J, we set constraints on the amounts of pre-existing CS dust that could account for the non-standard reddening measured by Goobar et al. (2014), Amanullah et al. (2014), Marion et al. (2015), Foley et al. (2014), Brown et al. (2015) and Ashall et al. (2014), who otherwise find this SN to be a normal SN Ia, albeit with slightly higher than average expansion velocities, and showing signs of additional sources of luminosity in the first hours after the explosion (Goobar et al. 2015). We also analyse other SNe Ia 


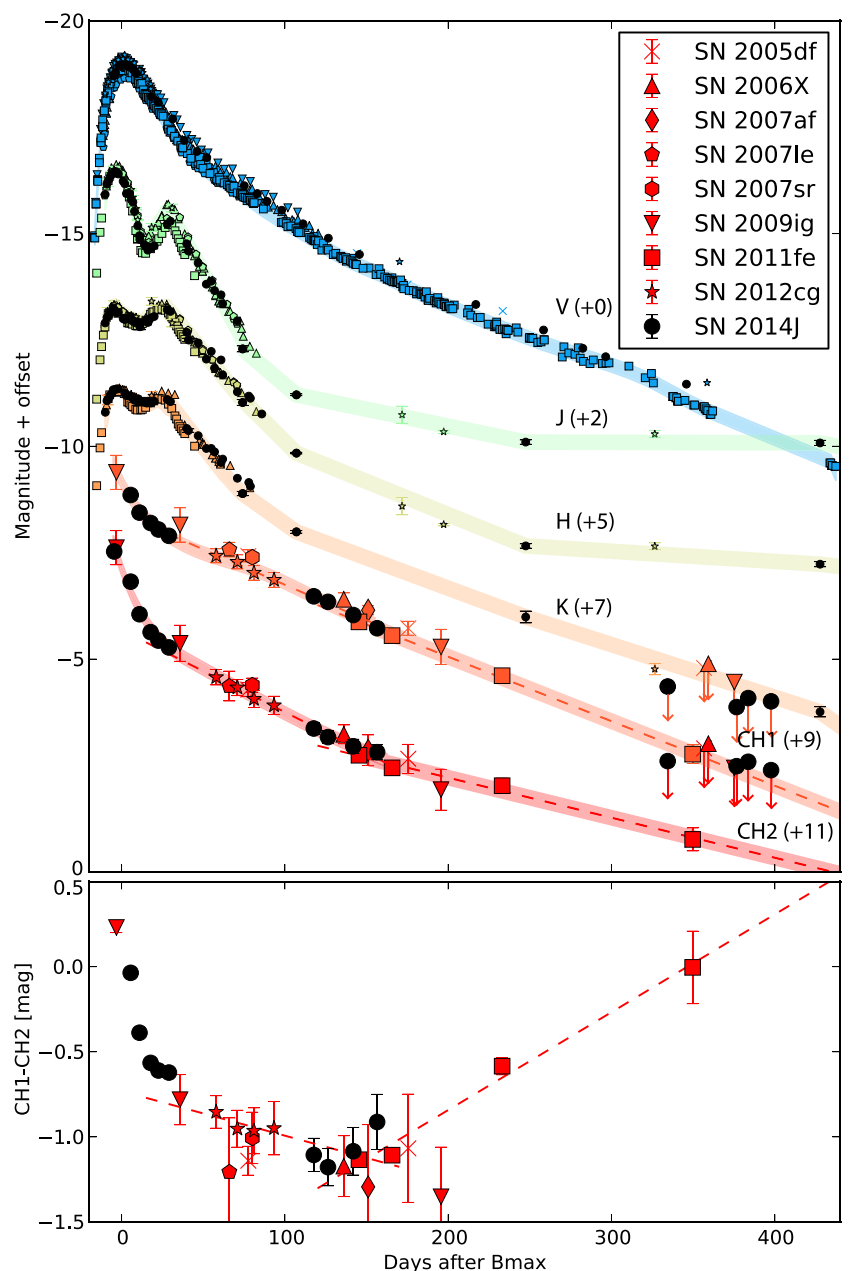

Figure 1. Upper panel: absolute magnitude $V$-band, near-IR $(J, H$ and $K$ band) and mid-IR light curves of the Type Ia SNe used in this study. The magnitudes have been de-reddened using the best-fitting extinction values and shifted by the distance moduli (typically known to $\sim 0.2$ mag accuracy) listed in Table 1. The lower panel shows the Spitzer $\mathrm{CH} 1-\mathrm{CH} 2$ colour evolution.

with peculiar reddening observed with Spitzer, SNe 2006X and 2007le, where CS material has been reported (Patat et al. 2007; Simon et al. 2009).

The outline of this paper is as follows: In Section 2, we present the observations, followed by a description in Section 3 of how the mid-IR light-curve templates are constructed. In Section 4, we add this new wavelength range along with optical and near-IR spectra, to present the spectral energy distribution (SED) in the full range 0.4-5.0 $\mu \mathrm{m}$. The implications for emission from heated CS dust are discussed in Section 5, followed by concluding remarks in Section 6.

\section{OBSERVATIONS}

\subsection{SN 2014J}

SN 2014J was observed with Spitzer over six epochs between January 28 and March 3 and four epochs between May 31 and July 8 under the SPitzer InfraRed Intensive Transients Survey (SPIRITS) program (PI: M. Kasliwal). SPIRITS is an ongoing IR survey that systematically searches 200 nearby galaxies for all types of transients and variables within a volume of $20 \mathrm{Mpc}$. Data are promptly processed with subtractions relative to archival images in the Spitzer Heritage Archive. The SPIRITS team undertakes a large concomitant ground-based survey in the optical and near-IR to characterize the Spitzer findings. For additional details about the survey and first discoveries, see Kasliwal et al. (2017).

Aperture photometry was performed at the location of the $\mathrm{SN}$ on the aligned Spitzer Post-Basic Calibrated Data for the SN and pre-SN images. Throughout the paper, we use the zero magnitude fluxes for the IRAC Channels 1 and $2(\mathrm{CH} 1$ and $\mathrm{CH} 2$, with central wavelengths of 3.6 and $4.5 \mu \mathrm{m}$, respectively) of $F_{\nu, 0}^{\mathrm{CH} 1}=280.9 \mathrm{Jy}$ and $F_{v, 0}^{\mathrm{CH} 2}=179.7 \mathrm{Jy}$. All photometry is listed Table 3 .

We also present new optical and near-IR spectra (summarized in Table 2) and photometry (to be published in a future paper) of SN 2014J in Figs 1 and 2.

\subsection{Comparison supernovae}

In order to analyse our mid-IR data on SN 2014J, we need to compare with other well-studied SNe Ia. For this study, we include SNe that have multi-epoch Spitzer data (see Table 3) and good optical/near-IR coverage. SN 2011fe was observed with Spitzer starting $145 \mathrm{~d}$ after $B$-band maximum. UV data (Mazzali et al. 2014) and optical to near-IR observations (Matheson et al. 2012; Pereira et al. 2013) together with high-resolution spectroscopy (Patat et al. 2013), show that SN 2011fe suffered little to no extinction, $E(B-V)=0.026 \pm 0.036$ mag, making it useful as a template of a pristine, normal SN Ia. We adopt a distance modulus to SN 2011fe of $\mu=28.93 \pm 0.16 \operatorname{mag}(D=6.1 \pm 0.45 \mathrm{Mpc})$ based on near-IR light curves from Matheson et al. (2012), which is in good agreement with the Cepheid distance in Mager, Madore \& Freedman (2013). SN 2012cg was observed with Spitzer (PI: A. Goobar) starting $58 \mathrm{~d}$ after $B$-band maximum. The $\mathrm{SN}$ shows signs of modest host galaxy reddening, with a colour excess of $E(B-V) \approx$ $0.2 \mathrm{mag}$, derived from both optical photometry and high-resolution spectroscopy (Silverman et al. 2012; Munari et al. 2013; Amanullah et al. 2015). Using optical light curves, Munari et al. (2013) put SN 2012cg at a distance $\mu=30.95$ mag, close to the TullyFisher estimate in Cortés, Kenney \& Hardy (2008). By accounting for the reddening and scaling the NIR photometry in Amanullah et al. (2015) to match SN 2011fe, we adopt a distance modulus of $\mu=30.70 \pm 0.16 \mathrm{mag}(D=13.8 \pm 1.0 \mathrm{Mpc})$.

To compare our limits on CS dust for SN 2014J, we also include archival data of the reddened SNe 2006X and 2007le. SN 2006X in M 100 was observed by Spitzer (PI's: P. Meikle and R. Kotak) starting $136 \mathrm{~d}$ after $B$-band maximum. Similar to SN 2014J, 2006X showed signs of non-standard reddening, $E(B-V) \sim 1.4$ mag with $R_{V} \sim 1.5$ (Wang et al. 2008a; Folatelli et al. 2010). SN 2007le suffered less extinction than SN 2006X, $E(B-V) \sim 0.39 \mathrm{mag}$, but also had a low $R_{V} \sim 1.5$ (Burns et al. 2014). The detection of time varying $\mathrm{Na}$ I $\mathrm{D}$ absorption for both these $\mathrm{SNe}$ has been interpreted as being due to CSM at distances $\sim 10^{17} \mathrm{~cm}$ from the SN (Patat et al. 2007; Simon et al. 2009). Since the SNe are reddened, it has been speculated that dust in the CS environment could play an important role. We also include data of SN 2005df and SN 2009ig previously presented in McClelland et al. (2013) and Gerardy et al. (2007), adding two epochs for SN 2009 ig at -3 and $+36 \mathrm{~d}$ from peak brightness, serendipitously observed with Spitzer (PI: K. Sheth). SN 2007af and SN 2007sr were observed with Spitzer (PI: R. Kotak) and have well-measured optical/near-IR light curves and precise Cepheid distance estimates (Riess et al. 2011). 


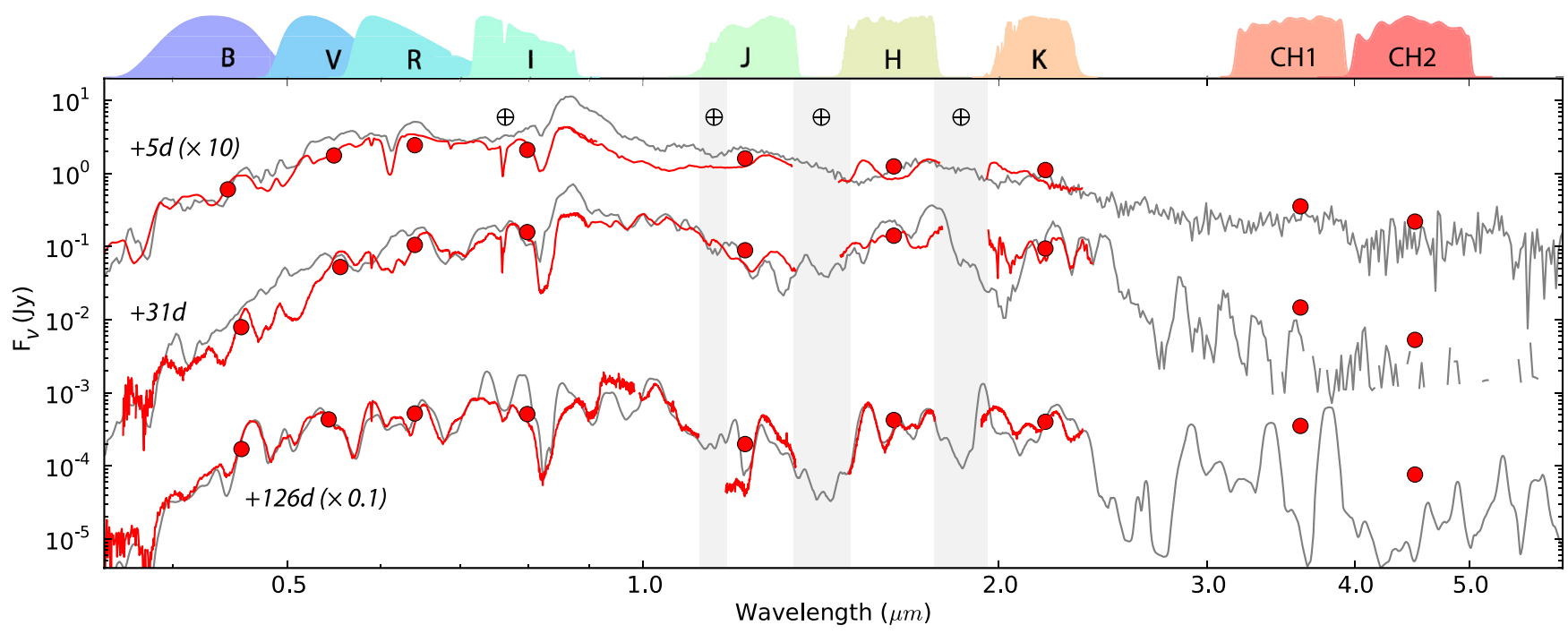

Figure 2. Observed SEDs on SN 2014J at $+5,+31$ and $+126 \mathrm{~d}$ past $B$-band maximum from optical/NIR spectroscopy (red lines) and broad-band BVRIJHK and Spitzer $\mathrm{CH} 1$ and $\mathrm{CH} 2$ photometry (red circles). For comparison, synthetic spectra of the W7 model (grey lines) at similar epochs are shown. Vertical, grey bars indicate regions of low atmospheric transmission.

Table 1. Type Ia supernovae observed in the mid-IR.

\begin{tabular}{|c|c|c|c|c|c|c|c|c|}
\hline Target & $\begin{array}{l}t_{B, \max } \\
\text { MJD }\end{array}$ & $\begin{array}{l}\text { Host } \\
\text { galaxy }\end{array}$ & $\begin{array}{c}\text { Distance } \\
\text { modulus (mag) }\end{array}$ & $\begin{array}{l}\text { Distance } \\
(\mathrm{Mpc})\end{array}$ & $\begin{array}{c}E(B-V) \\
\quad(\mathrm{mag})\end{array}$ & $R_{V}$ & $\begin{array}{l}\Delta m_{15}^{B} \\
(\mathrm{mag})\end{array}$ & Reference \\
\hline $2005 \mathrm{df}$ & 53598.4 & NGC 1559 & $31.26(0.14)$ & $15.7(2.3)$ & 0.03 & 3.1 & 1.20 & $1,2,3$ \\
\hline $2006 X$ & 53785.6 & NGC 4321 & $30.91(0.14)$ & $15.2(1.0)$ & 1.24 & 1.6 & 1.29 & 4,5 \\
\hline 2007af & 54173.8 & NGC 5584 & $31.72(0.07)$ & $22.0(0.7)$ & 0.04 & 3.0 & 1.20 & $6,7,8$ \\
\hline 2007le & 54398.9 & NGC 7721 & $32.24(0.16)$ & $28.1(2.1)$ & 0.39 & 1.5 & 1.10 & 9 \\
\hline $2007 \mathrm{sr}$ & 54448.3 & NGC 4038 & $31.66(0.08)$ & $21.5(0.8)$ & 0.13 & 1.6 & 0.97 & 6,10 \\
\hline 2009ig & 55080.9 & NGC 1015 & $32.82(0.09)$ & $36.6(1.5)$ & $<0.05$ & - & 0.89 & $2,11,12$ \\
\hline $2011 \mathrm{fe}$ & 55815.8 & NGC 5457 & $28.93(0.16)$ & $6.1(0.5)$ & 0.03 & 3.1 & 1.11 & $2,13,14$ \\
\hline $2012 \mathrm{cg}$ & 56082.0 & NGC 4424 & $30.70(0.16)$ & $13.8(1.0)$ & 0.15 & 2.6 & 0.89 & $14,15,16$ \\
\hline $2014 \mathrm{~J}$ & 56689.2 & M82 & $27.60(0.16)$ & $3.3(0.15)$ & 1.37 & 1.4 & 0.95 & $17,18,19$ \\
\hline
\end{tabular}

1: Gerardy et al. (2007), 2: McClelland et al. (2013), 3: Milne et al. (2010), 4: Wang et al. (2008a), 5: Folatelli et al. (2010), 6: Riess et al. (2011), 7: Simon et al. (2007), 8: Hicken et al. (2009), 9: Burns et al. (2014), 10: Schweizer et al. (2008), 11: Foley et al. (2012), 12: Marion et al. (2013), 13: Matheson et al. (2012), 14: Munari et al. (2013), 15: Silverman et al. (2012), 16: Amanullah et al. (2015), 17: Amanullah et al. (2014), 18: Foley et al. (2014), 19: Marion et al. (2015), 20: Amanullah et al. (2015).

Table 2. Log of spectroscopic observations of SN 2014J.

\begin{tabular}{lcccccc}
\hline UT date & MJD & $\begin{array}{c}\text { Days from } \\
B_{\max }\end{array}$ & $\begin{array}{c}\text { Observatory/ } \\
\text { instrument }\end{array}$ & $\begin{array}{c}\text { Wavelength range } \\
(\mu \mathrm{m})\end{array}$ & $\begin{array}{c}\text { Exp. time } \\
(\mathrm{s})\end{array}$ \\
\hline $2014-02-06^{a}$ & 56694.9 & +5.1 & NOT/ALFOSC & $0.35-0.90$ & 180 & 1.61 \\
$2014-02-06^{a}$ & 56695.0 & +5.2 & Mt Abu & $0.85-2.35$ & 1200 & 1.59 \\
$2014-03-03$ & 56718.9 & +29.1 & Mt Abu & $0.85-2.35$ & 1080 & 1.48 \\
$2014-03-05$ & 56721.1 & +31.3 & NOT/ALFOSC & $0.35-0.90$ & 1.30 & 1.35 \\
$2014-06-07$ & 56815.3 & +125.5 & Keck/MOSFIRE & $1.15-2.35$ & 480 \\
$2014-06-09$ & 56817.2 & +127.4 & ARC/DIS & $0.35-0.95$ & 120 \\
\hline
\end{tabular}

Note. ${ }^{a}$ Published in Marion et al. (2015).

\section{MID-IR LIGHT CURVES}

SN 2014J is the best object to date to build mid-IR light-curve templates, capturing the full range from before peak brightness to the nebular phase. To fill in the gaps, caused by limited visibility windows and scheduling constraints, we make use of archival data of the SNe Ia described in Section 2.2. The composite light curves shown in Fig. 1 are compiled by shifting each SN by their estimated distance modulus and correcting for host galaxy and Milky Way extinction (see Table 1). We do not find evidence for variations in the mid-IR light-curve shapes corresponding to the different optical decline rates in our sample, $\Delta m_{15}^{B}=0.9-1.3$, suggesting that $\mathrm{SNe}$ Ia are a very homogeneous class of objects at longer wavelengths. A larger sample of SNe Ia with multi-epoch mid-IR coverage is needed to make a more quantitative study. Three different decline time-scales can be recognized in the $\mathrm{CH} 1$ and $\mathrm{CH} 2$ light curves. Although our first detections are before the optical and near-IR maximum brightness, we cannot fully measure the mid-IR lightcurve shapes at these epochs, i.e. fitting the time of maximum in 
Table 3. Spitzer observations and photometry of Type Ia supernovae in the mid-IR.

\begin{tabular}{|c|c|c|c|c|c|c|c|}
\hline SN & MJD & $\begin{array}{l}\text { Epoch } \\
\text { (d) }\end{array}$ & $\begin{array}{l}F_{v}^{\mathrm{CH} 1} \\
(\mu \mathrm{Jy})\end{array}$ & $\begin{array}{l}F_{v}^{\mathrm{CH} 2} \\
(\mu \mathrm{Jy})\end{array}$ & $\begin{array}{c}\text { CH1 } \\
\text { (mag) }\end{array}$ & $\begin{array}{c}\mathrm{CH} 2 \\
\text { (mag) }\end{array}$ & $\begin{array}{l}\text { Sub? } \\
(\mathrm{y} / \mathrm{n})\end{array}$ \\
\hline $2005 \mathrm{df}$ & 53676.0 & 77.6 & $327.0(6.7)$ & $73.2(5.7)$ & $14.835(0.022)$ & $15.976(0.082)$ & $\mathrm{y}$ \\
\hline $2005 \mathrm{df}$ & 53774.0 & 175.6 & $68.3(5.6)$ & $16.4(5.3)$ & $16.535(0.085)$ & $17.602(0.307)$ & $\mathrm{y}$ \\
\hline $2005 \mathrm{df}$ & 53955.1 & 356.7 & $13.3(5.2)$ & $4.6(5.3)$ & $>18.136$ & $>17.642$ & $\mathrm{y}$ \\
\hline $2006 X$ & 53922.2 & 136.1 & $157.9(8.0)$ & $35.3(6.0)$ & $15.626(0.054)$ & $16.768(0.171)$ & $\mathrm{y}$ \\
\hline $2006 X$ & 54145.4 & 359.3 & $19.7(8.0)$ & $13.3(6.0)$ & $>17.750$ & $>17.572$ & $\mathrm{y}$ \\
\hline $2006 X$ & 54662.7 & 876.6 & $-0.7(7.6)$ & $1.8(5.9)$ & $>17.731$ & $>17.520$ & $\mathrm{y}$ \\
\hline $2006 X$ & 56722.8 & 2936.7 & $-6.9(8.3)$ & $-1.3(6.5)$ & $>17.633$ & $>17.415$ & $\mathrm{y}$ \\
\hline $2006 X$ & 56751.9 & 2965.8 & $1.6(8.6)$ & $0.2(6.3)$ & $>17.586$ & $>17.447$ & $\mathrm{y}$ \\
\hline 2007af & 54324.9 & 151.1 & $62.2(4.6)$ & $12.8(5.0)$ & $16.594(0.081)$ & $17.865(0.357)$ & $\mathrm{y}$ \\
\hline 2007af & 55280.4 & 1106.6 & $2.4(5.0)$ & $2.2(5.0)$ & $>18.181$ & $>17.696$ & $\mathrm{y}$ \\
\hline 2007le & 54464.7 & 66.0 & $152.0(5.0)$ & $32.0(5.0)$ & $15.667(0.059)$ & $16.873(0.312)$ & $\mathrm{y}$ \\
\hline $2007 \mathrm{sr}$ & 54528.1 & 80.1 & $220.0(8.0)$ & $55.7(10.0)$ & $15.265(0.146)$ & $16.271(0.146)$ & $\mathrm{y}$ \\
\hline $2007 \mathrm{sr}$ & 55616.4 & 1168.4 & $3.3(8.0)$ & $19.2(8.0)$ & $>17.671$ & $>17.186$ & $\mathrm{y}$ \\
\hline 2009ig & 55076.9 & -3.1 & $580.7(10.0)$ & $459.1(10.0)$ & $14.211(0.019)$ & $13.982(0.023)$ & $\mathrm{n}$ \\
\hline 2009ig & 55115.9 & 35.8 & $186.1(8.0)$ & $57.9(8.0)$ & $15.447(0.046)$ & $16.229(0.140)$ & $\mathrm{n}$ \\
\hline 2009ig & 55275.7 & 195.6 & $13.3(1.4)$ & $2.4(0.7)$ & $18.316(0.110)$ & $19.668(0.270)$ & $\mathrm{n}$ \\
\hline 2009ig & 55455.2 & 375.2 & $2.9(1.1)$ & $1.5(0.8)$ & $>19.876$ & $>19.672$ & $\mathrm{n}$ \\
\hline $2011 \mathrm{fe}$ & 55960.7 & 145.4 & $670.6(8.5)$ & $150.9(6.4)$ & $14.055(0.014)$ & $15.190(0.045)$ & $\mathrm{y}$ \\
\hline $2011 \mathrm{fe}$ & 55981.0 & 165.6 & $498.2(8.1)$ & $114.9(6.3)$ & $14.378(0.018)$ & $15.486(0.058)$ & $\mathrm{y}$ \\
\hline $2011 \mathrm{fe}$ & 56048.4 & 233.0 & $209.2(7.0)$ & $78.1(5.7)$ & $15.320(0.036)$ & $15.905(0.076)$ & $\mathrm{y}$ \\
\hline $2011 \mathrm{fe}$ & 56165.0 & 349.7 & $38.3(5.7)$ & $24.4(5.4)$ & $17.163(0.151)$ & $17.168(0.217)$ & $\mathrm{y}$ \\
\hline $2011 \mathrm{fe}$ & 56337.1 & 521.8 & $3.3(5.6)$ & $14.1(6.1)$ & $>18.058$ & $17.763(0.390)$ & $\mathrm{y}$ \\
\hline $2011 \mathrm{fe}$ & 56348.1 & 532.8 & $2.5(5.8)$ & $15.6(5.0)$ & $>18.020$ & $17.654(0.302)$ & $\mathrm{y}$ \\
\hline $2011 \mathrm{fe}$ & 56393.8 & 578.5 & $4.4(5.1)$ & $7.8(7.7)$ & $>18.160$ & $>17.227$ & $\mathrm{y}$ \\
\hline $2011 \mathrm{fe}$ & 56452.6 & 637.3 & $3.9(6.0)$ & $-2.6(4.6)$ & $>17.983$ & $>17.787$ & $\mathrm{y}$ \\
\hline $2011 \mathrm{fe}$ & 56742.8 & 927.5 & - & $2.4(5.7)$ & - & $>17.554$ & $\mathrm{y}$ \\
\hline $2011 \mathrm{fe}$ & 56771.8 & 956.5 & - & $1.9(5.8)$ & - & $>17.534$ & $\mathrm{y}$ \\
\hline $2011 \mathrm{fe}$ & 56902.0 & 1086.7 & $3.1(7.4)$ & $-8.9(11.2)$ & $>17.755$ & $>16.820$ & $\mathrm{y}$ \\
\hline $2012 \mathrm{cg}$ & 56139.9 & 57.9 & $549.0(20.3)$ & $160.0(13.3)$ & $14.272(0.039)$ & $15.126(0.087)$ & $\mathrm{y}$ \\
\hline $2012 \mathrm{cg}$ & 56152.9 & 70.9 & $481.0(19.0)$ & $128.0(12.3)$ & $14.416(0.042)$ & $15.368(0.100)$ & $\mathrm{y}$ \\
\hline $2012 \mathrm{cg}$ & 56163.1 & 81.1 & $380.0(20.4)$ & $100.0(11.9)$ & $14.672(0.057)$ & $15.636(0.122)$ & $\mathrm{y}$ \\
\hline $2012 \mathrm{cg}$ & 56175.4 & 93.4 & $326.0(20.6)$ & $87.0(12.2)$ & $14.838(0.067)$ & $15.788(0.142)$ & $\mathrm{y}$ \\
\hline $2012 \mathrm{cg}$ & 56723.8 & 641.8 & $11.9(17.2)$ & $6.2(12.6)$ & $>16.837$ & $>16.694$ & $\mathrm{y}$ \\
\hline $2012 \mathrm{cg}$ & 56751.9 & 669.9 & $4.1(18.4)$ & $2.2(12.7)$ & $>16.764$ & $>16.682$ & $\mathrm{y}$ \\
\hline $2014 \mathrm{~J}$ & 56685.4 & -4.5 & - & $42273.0(200.0)$ & - & $9.071(0.005)$ & $\mathrm{y}$ \\
\hline $2014 J$ & 56695.4 & 5.6 & 35895.9 (154.9) & $22130.1(97.1)$ & $9.734(0.005)$ & $9.774(0.005)$ & $\mathrm{y}$ \\
\hline $2014 J$ & 56700.8 & 11.0 & $24508.9(149.2)$ & $10554.5(95.9)$ & $10.148(0.007)$ & $10.578(0.010)$ & $\mathrm{y}$ \\
\hline $2014 \mathrm{~J}$ & 56707.6 & 17.8 & 18295.8 (117.4) & $7130.0(73.1)$ & $10.466(0.007)$ & $11.004(0.011)$ & $\mathrm{y}$ \\
\hline $2014 J$ & 56712.4 & 22.6 & $16491.0(121.1)$ & $5932.9(75.2)$ & $10.578(0.008)$ & $11.203(0.014)$ & $\mathrm{y}$ \\
\hline $2014 J$ & 56718.8 & 29.0 & $14325.6(129.4)$ & $5145.3(80.5)$ & $10.731(0.010)$ & $11.358(0.017)$ & $\mathrm{y}$ \\
\hline $2014 J$ & 56807.5 & 117.7 & $3812.2(127.3)$ & $876.4(80.3)$ & $12.168(0.036)$ & $13.280(0.095)$ & $\mathrm{y}$ \\
\hline $2014 J$ & 56816.2 & 126.4 & $3273.6(122.5)$ & $766.0(75.8)$ & $12.334(0.040)$ & $13.426(0.102)$ & $\mathrm{y}$ \\
\hline $2014 \mathrm{~J}$ & 56831.7 & 141.9 & $2338.2(121.3)$ & $600.4(79.9)$ & $12.699(0.055)$ & $13.690(0.136)$ & $\mathrm{y}$ \\
\hline $2014 \mathrm{~J}$ & 56846.3 & 156.5 & $1922.3(126.5)$ & $448.6(79.9)$ & $12.912(0.069)$ & $14.007(0.178)$ & $\mathrm{y}$ \\
\hline $2014 J$ & 57024.2 & 334.4 & $203.3(130.2)$ & $212.6(77.6)$ & $>14.482$ & $>13.997$ & $\mathrm{y}$ \\
\hline $2014 \mathrm{~J}$ & 57066.4 & 376.6 & $1.3(131.8)$ & $165.7(79.3)$ & $>14.601$ & $>14.116$ & $\mathrm{y}$ \\
\hline $2014 J$ & 57073.5 & 383.7 & $78.7(132.1)$ & $206.2(79.4)$ & $>14.498$ & $>14.013$ & $\mathrm{y}$ \\
\hline $2014 \mathrm{~J}$ & 57087.5 & 397.7 & $48.8(132.0)$ & $132.3(79.3)$ & $>14.694$ & $>14.209$ & $\mathrm{y}$ \\
\hline
\end{tabular}

$\mathrm{CH} 1$ and $\mathrm{CH} 2$ is impossible. However, fitting the early epochs ( -5 to $15 \mathrm{~d}$ from $B$-band maximum) gives linear decline rates of 0.081 and $0.135 \mathrm{mag} \mathrm{d}^{-1}$ in $\mathrm{CH} 1$ and $\mathrm{CH} 2$, respectively, similar to the decline rates in optical bands.

A break in the mid-IR light curves occurs $\sim 15 \mathrm{~d}$ after $B$ band maximum. This roughly coincides with the onset of the secondary maximum in the near-IR bands, although data from +15 to $+30 \mathrm{~d}$ show no signs of a secondary maximum in the midIR. From $15 \mathrm{~d}$ after $B$-band maximum and onwards, the decline rate changes to 1.67 and $1.93 \mathrm{mag} / 100 \mathrm{~d}$ in $\mathrm{CH} 1$ and $\mathrm{CH} 2$, respectively. After $\sim 150 \mathrm{~d}$ past $B$-band maximum the linear decline rates of SN $2011 \mathrm{fe}$ are 1.48 and $0.78 \mathrm{mag} / 100 \mathrm{~d}$ in $\mathrm{CH} 1$ and $\mathrm{CH} 2$, respectively. The decline rate in $\mathrm{CH} 1$ is similar to what is observed at optical wavelengths, while the decline in $\mathrm{CH} 2$ is slower, which can also be seen in the $\mathrm{CH} 1-\mathrm{CH} 2$ colour panel in Fig. 1 as a change towards redder colours. McClelland et al. (2013) analyse the mid-IR late-time ( $>200 \mathrm{~d}$ ) decline rates for four SNe (SN 2011fe, SN 2009ig, SN 2008Q and SN 2005df) and argue that 
the different decline rates are a result of doubly ionized elements dominating the bluer $\mathrm{CH} 1$ band $(3.6 \mu \mathrm{m})$ while singly ionized iron-peak species are controlling the redder $\mathrm{CH} 2$ band $(4.5 \mu \mathrm{m})$. They also suggest that the interpolated colour at $+230 \mathrm{~d}$ correlates with the light-curve decline rate at maximum brightness, i.e. with $\Delta m_{15}^{B}$.

\section{SPECTRAL ENERGY DISTRIBUTION}

Lacking spectra in the wavelength range probed by our Spitzer observations, we examine how the shape of the SED, as measured through optical/near-IR spectroscopy together with the Spitzer broad-band observations, matches model spectra from numerical simulations. In Fig. 2, we show observed optical and near-IR spectra (red lines) together with optical, near-IR and mid-IR broad-band photometry (red circles) of SN 2014J at three epochs (5, 31 and $126 \mathrm{~d}$ after $B$-band maximum).

To this end, we have calculated synthetic spectra for the hydrodynamic explosion model W7 (Nomoto, Thielemann \& Yokoi 1984). W7 is known to reproduce the observed characteristics of normal SNe Ia at optical (e.g. Branch et al. 1985) and near-IR wavelengths (e.g. Gall et al. 2012). To obtain synthetic spectra at 5 and $31 \mathrm{~d}$ past $B$-band maximum, we performed radiative transfer simulations using the ARTIS code (Kromer \& Sim 2009) and the atomic data set described in Gall et al. (2012). For the latest epoch (+126 d past $B$-band maximum), we used a nebular code (described in Jerkstrand, Fransson \& Kozma 2011; Jerkstrand et al. 2015). The flux in the model spectra between $B$-band maximum and $+10 \mathrm{~d}$ stems from a mixture of $\mathrm{Co}$ II, $\mathrm{Co}$ III and $\mathrm{Fe}$ II features and a tiny contribution from intermediate-mass elements. Starting at $10 \mathrm{~d}$ after maximum, the flux in the 2.8-3.5 micron region is dominated by singly ionized iron-group elements. The lines in the W7 model possibly contributing to the flux in $\mathrm{CH} 1$ can be attributed to $\mathrm{Ni}$ II (at 2.85, 2.95, 3.11, 3.29 and $3.54 \mu \mathrm{m})$ and $\mathrm{Fe}$ II $(3.08 \mu \mathrm{m})$.

In the late-time model spectrum $(+126 \mathrm{~d}$ after maximum), the fluxes in the optical and near-IR agree with the observations while the flux levels in the mid-IR are underpredicted. The dominant lines in $\mathrm{CH} 1$ and $\mathrm{CH} 2$ are [Fe II], with little contribution from [Co II]. In $\mathrm{CH} 1$, there is also [Fe III] (at 2.90 and $3.04 \mu \mathrm{m}$ ) and [Co III] (at $3.48 \mu \mathrm{m}$ ) emission.

Our observations, shown in Figs 1 and 2, bridge the gap between the late-time near-IR spectra in Friesen et al. (2014) and mid-IR spectra of SN 2014J in the 8-13 $\mu \mathrm{m}$ wavelength range in Telesco et al. (2015). Friesen et al. (2014) present near-IR spectra of SN $2014 \mathrm{~J}$ at $70 \mathrm{~d}$ past maximum and find that [Ni II] fits the emission feature near $1.98 \mu \mathrm{m}$, suggesting that a substantial mass of ${ }^{58} \mathrm{Ni}$ exists near the centre of the ejecta, arising from nuclear burning at high density. A tentative identification of $\mathrm{Mn}$ II at $1.15 \mu \mathrm{m}$ may support this conclusion as well. Telesco et al. (2015) compare their observed mid-IR spectra to a delayed detonation model with $\sim 0.6 \mathrm{M}_{\odot}$ of ${ }^{56} \mathrm{Ni}$ and claim that the model is consistent with observations. Recent multidimensional hydrodynamical simulations of Chandrasekhar-mass explosions, however, struggle in producing a concentration of stable iron-group elements near the centre of the ejecta (e.g. Seitenzahl et al. 2013).

\section{CONSTRAINTS ON EMISSION FROM CS DUST}

The existence of CS material around individual nearby SNe Ia has been suggested by studies of sodium absorption lines (e.g. SNe 1999cl, 2006X, 2007le, and PTF11kx, Patat et al. 2007;
Blondin et al. 2009; Simon et al. 2009; Dilday et al. 2012). High-resolution spectra reveal the presence of time-variable and blueshifted $\mathrm{Na}$ I D features, possibly originating from CSM within the progenitor system. Studies of large samples of SNe Ia (Sternberg et al. 2011) find that half of all SNe Ia with detectable Na I D absorption at the host-galaxy redshift have $\mathrm{Na}$ I D line profiles with significant blueshifted absorption relative to the strongest absorption component. This indicates that the absorption occurs in the vicinity of the progenitor systems rather than in the ISM. For SN 2014J, high-resolution spectra reveal no signs of time-variable Na I D absorption (Foley et al. 2014; Goobar et al. 2014; Welty et al. 2014; Ritchey et al. 2015; Maeda et al. 2016), consistent with a clean CS environment within $\sim 10^{17} \mathrm{~cm}$. However, Graham et al. (2015) find time-variable $\mathrm{K}$ I, which might be due to ionization of material at even larger distances $\left(\sim 10^{19} \mathrm{~cm}\right)$. The wavelength-dependent extinction towards SN 2014J has been measured with great accuracy using a very wide wavelength range, $0.2-2.2 \mu \mathrm{m}$, through a combination of Hubble Space Telescope and ground-based observations. Amanullah et al. (2014) found that the reddening can be described with either an MW-type extinction law with a low value of the total-to-selective extinction, $R_{V}=1.4 \pm 0.1$, i.e. corresponding to non-standard dust grains in the ISM, or by invoking the effective extinction law from Goobar (2008). The latter arises from multiple scattering of photons on 'normal' dust grains, but surrounding the supernova, i.e. in the CS medium, as also discussed by Wang (2005).

Non-standard reddening has been noted in studies of individual and large samples of SNe Ia. For example, the extinction of SN 2006X was studied in Folatelli et al. (2010), showing that the reddening is incompatible with the average extinction law of the Milky Way. Their findings augmented the large body of evidence indicating that the reddening of many SNe Ia show a steeper wavelength dependence $\left(R_{V}<3.1\right)$ than that which is typically observed for stars in our Galaxy. Previously, Nobili \& Goobar (2008) derived $R_{V}=1.75 \pm 0.27$ from a statistical study of 80 low-redshift $\mathrm{SNe}$ Ia. Similarly, when the colour-brightness relation is fitted jointly with cosmological parameters in the SN Ia Hubble diagram, using a wide range of SN Ia redshifts, low values of $R_{V}$ are obtained (see e.g. Betoule et al. 2014, for a recent compilation).

Amanullah \& Goobar (2011) simulated the impact of thin CS dust shells located at radii $r_{\mathrm{d}} \sim 10^{16}-10^{19} \mathrm{~cm}(\sim 0.003-3 \mathrm{pc})$ from the $\mathrm{SN}$ and found that this scenario would also perturb the optical light-curve shapes and introduce a time-dependent colour excess, $\Delta E(B-V) \sim 0.05-0.1$ mag. Foley et al. (2014) claim to have detected a time-variable colour excess for SN 2014J, which led them to conclude that dimming by CS dust accounts for about half of the extinction. However, this interpretation has been challenged by Brown et al. (2015). By exploring the mid-IR wavelength range, we have a unique way to test if dust in the CSM plays a significant role in explaining the non-standard reddening towards SN 2014J and other highly reddened normal SNe Ia.

\subsection{Dust models}

If pre-existing CS dust is the source of non-standard reddening, it will be radiatively heated by absorption of UV/optical photons from the SN or collisionally heated by the SN shock. Thermal emission at IR wavelengths could therefore be the 'smoking gun' for detecting or ruling out the presence of CS dust. To model the emission from pre-existing CS dust, we consider the idealized case (described in Hildebrand 1983; Dwek 1985; Fox et al. 2010) of an optically thin (at mid-IR wavelengths) dust cloud of mass $M_{\mathrm{d}}$ with dust particles 


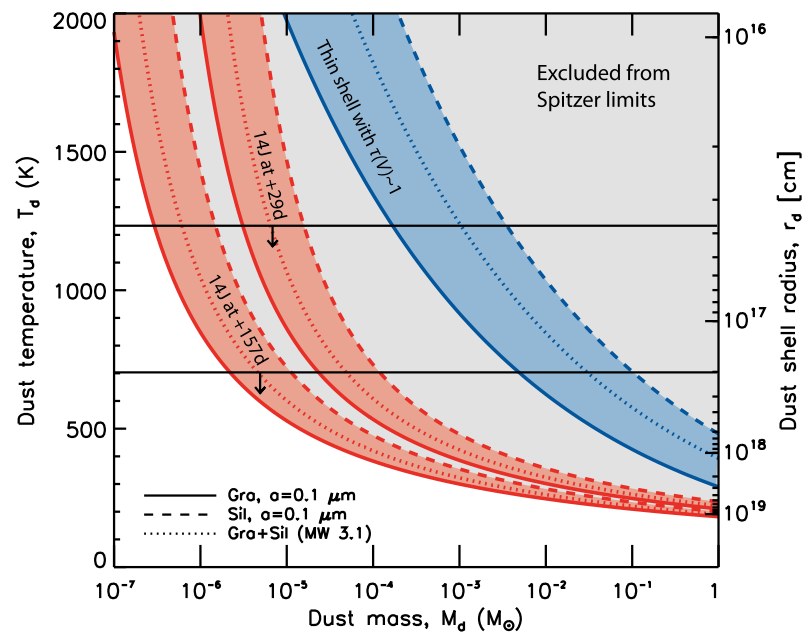

Figure 3. The red lines show $3 \sigma$ upper limits on dust emission around $\mathrm{SN}$ 2014J using Spitzer $4.5 \mu \mathrm{m}$ data at 29 and $157 \mathrm{~d}$ after maximum brightness. The solid, dashed and dotted contours indicate limits using the graphitic, silicate and MW3.1 dust models, respectively. Assuming the thin shell approximation, we can estimate the expected dust temperatures and shell radii probed at these epochs (black lines) and the expected dust mass from equation (3) (blue lines).

of radius $a$, emitting thermally at a single equilibrium temperature $T_{\mathrm{d}}$. The expected flux at a distance $D$ is

$F_{v}=M_{\mathrm{d}} \frac{\kappa_{v}(a) B_{v}\left(T_{\mathrm{d}}\right)}{D^{2}}$,

where $B_{v}\left(T_{\mathrm{d}}\right)$ is the Planck blackbody function and $\kappa_{v}(a)$ is the dust mass absorption coefficient.

Since we do not know the nature of the SN Ia progenitor systems and their potential dust production mechanisms, we will consider separate scenarios of either silicate or graphite grains of radius $a=0.1 \mu \mathrm{m}$ and a mixture of silicate and graphitic grains of different sizes (MW3.1) that reproduce the standard $R_{V}=3.1$ Milky Way dust properties (described in Draine \& Lee 1984; Laor \& Draine 1993; Weingartner \& Draine 2001).

In what follows, we assume that the low-reddening SNe $2011 \mathrm{fe}$ and 2012 cg represent the intrinsic IR flux from an SN Ia (dashed lines in Fig. 1), to put limits on any additional excess emission from CS dust around the reddened SNe 2014J, 2006X and 2007le. Taking both instrumental noise and distance modulus uncertainties into account, the differences between the reddened and unreddened $\mathrm{SNe}$ are not statistically significant. Hence, we compute $3 \sigma$ upper limits on $F_{\text {dust }}=F_{\text {redSN }}-F_{\text {unred.SN }}$ from the Spitzer data, and use equation (1) to constrain the dust temperature and mass for SN 2014J $(4.5 \mu \mathrm{m}$ limits shown as red contours in Fig. 3) as well as for other $\mathrm{SNe}(4.5 \mu \mathrm{m}$ limits shown as red contours in Fig. 4). The $3.6 \mu \mathrm{m}$ limits are comparable, but slightly less constraining than those obtained from the $4.5 \mu$ data. We complement this with a similar analysis using $K$-band data of SNe 2014J and 2006X (green contours in Fig. 4).

\subsection{Expected emission from heated dust in the thin shell approximation}

In order to break the degeneracy in the $M_{\mathrm{d}}-T_{\mathrm{d}}$ plane, we make some further assumptions on the dust model. Although the geometric distribution of CS dust could be complex, we adopt the simple thin shell approximation where the dust is distributed uniformly within

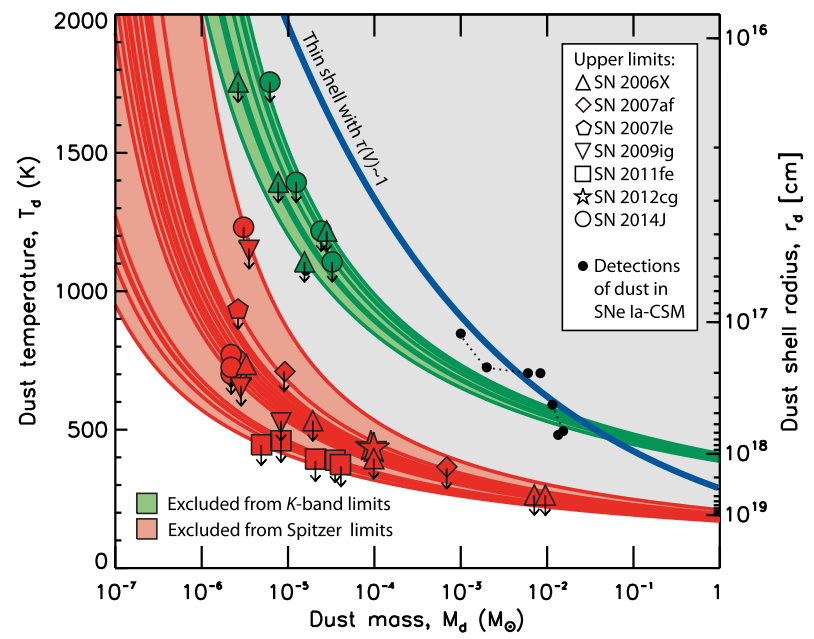

Figure 4. $3 \sigma$ upper limits on CS dust emission around the reddened $\mathrm{SNe}$ 2014J, 2006X and 2007le at different epochs from $K$-band data (green contours and symbols) and Spitzer $4.5 \mu \mathrm{m}$ data (red contours and symbols), assuming graphitic dust gains of radius $0.1 \mu \mathrm{m}$. Also shown are the Spitzer limits for SNe 2007af and 2009ig along with the late time non-detections of SNe $2011 \mathrm{fe}$ and $2012 \mathrm{cg}$. The blue solid line indicates the expected dust mass from equation (3). Black dots are detections of warm dust around Ia-CSM $\mathrm{SNe}$.

the shell, to provide an estimate of the expected mid-IR emission from heated dust (comparable to the models for reddening proposed in Goobar 2008; Amanullah \& Goobar 2011). This allows us to estimate the expected temperature as a function of shell radius, shown as the right-hand side vertical axis of Figs 3 and 4 . We estimate the minimal dust shell radius, $r_{\mathrm{d}}=c t / 2$, that could give rise to a detectable IR echo at each observed epoch, $t$. Dust at this radius will be heated to

$T_{\mathrm{d}, \exp } \sim 4.0\left(\frac{L}{a}\right)^{\frac{1}{6}} r_{\mathrm{d}}^{-\frac{1}{3}}$,

where we assume that the peak $\mathrm{SN}$ bolometric luminosity of $\sim 3 \times 10^{9} \mathrm{~L}_{\odot}$ is heating a spherical dust shell with grain size of $a=0.1 \mu \mathrm{m}$ (Kruegel 2003). The upper bound on the dust temperature is set by the evaporation temperature of the dust grains ( $T \lesssim 2000 \mathrm{~K}$ ), corresponding to a minimal dust survival radius $r_{\text {evap }} \sim 10^{16} \mathrm{~cm}$.

Furthermore, in order for a thin dust shell at $r_{\mathrm{d}}$ to have significant opacity in the optical $V$-band, $\tau(V) \sim 1$, the required dust mass can be estimated from the absorption cross-sections, $\sigma_{\text {abs }}(\lambda=0.55 \mu \mathrm{m}) / m_{\text {dust }}=\kappa_{V}$, as

$M_{\mathrm{d}, \exp } \sim 4 \pi r_{\mathrm{d}}^{2} \frac{\tau(V)}{\kappa(V)}$,

where $\kappa(V) \sim 5 \cdot 10^{4} \mathrm{~cm}^{2} \mathrm{~g}^{-1}$ for graphitic grains, $\kappa(V) \sim 2$. $10^{3} \mathrm{~cm}^{2} \mathrm{~g}^{-1}$ for silicate grains of size $a=0.1 \mu \mathrm{m}$ and $\kappa(V) \sim$ $2 \cdot 10^{3} \mathrm{~cm}^{2} \mathrm{~g}^{-1}$ for the MW3.1 mixture. Thus, for a thin spherical dust shell at $r_{\mathrm{d}}$ the total dust mass, $M_{\mathrm{d} \text {, exp }} \sim 10^{-4}$ $10^{-5}\left(r_{\mathrm{d}} / 10^{16} \mathrm{~cm}\right)^{2} \mathrm{M}_{\odot}$, depending on dust grain composition, is needed to explain the observed reddening if it mainly arises in the CS environment. This corresponds to the blue lines in Figs 3 and 4. The black horizontal lines in Fig. 3 indicate the expected dust temperature (and corresponding shell radius) at two of the epochs (29 and $157 \mathrm{~d}$ from $B$-band maximum) from where the limits are derived for SN 2014J. Similarly, the symbols in Fig. 4 indicate the limits 
derived at different epochs for an extended sample of $\mathrm{SNe}$ observed Spitzer.

Using $K$-band data for SNe 2014J and 2006X, to explore possible emission from hot CS dust close to the exploding $\operatorname{star}\left(T_{\mathrm{d}} \gtrsim 1200 \mathrm{~K}\right.$ for $r_{\mathrm{d}}<5 \cdot 10^{16} \mathrm{~cm}$ ), we are able to rule out $M_{\mathrm{d}}>10^{-5} \mathrm{M}_{\odot}$. These limits are comparable to the results in Maeda et al. (2015). By adding the Spitzer data, we can significantly reduce the allowed parameter space, excluding $M_{\mathrm{d}}>10^{-6} \mathrm{M}_{\odot}\left(T_{\mathrm{d}}>700 \mathrm{~K}\right.$, for $r_{\mathrm{d}}<$ $\left.2 \cdot 10^{17} \mathrm{~cm}\right)$.

Our combined limits for SNe 2014J correspond to an upper limit on the possible amount of pre-existing dust surrounding the $\mathrm{SN}$ progenitor, $M_{\mathrm{d}} \lesssim 10^{-5} \mathrm{M}_{\odot}$ within $r_{\mathrm{d}} \sim 2 \cdot 10^{17} \mathrm{~cm}$, depending on the assumed grain size and composition. However, regardless of the specific dust grain composition, the derived upper limits on the CS dust mass are significantly lower than what would be required to explain the observed reddening of SN 2014J, in apparent contraction to the claims in Foley et al. (2014). Similar limits are obtained for the reddened SNe 2006X and 2007le (see Fig. 4). For reference, we also show detections of dust around a subset of peculiar $\mathrm{SNe}$ Ia interacting strongly with a dense CSM (SNe Ia-CSM) in Fig. 4. Spitzer observations of SNe Ia-CSM 2002ic, 2005gj and 2008J (Fox et al. 2011; Taddia et al. 2012; Fox \& Filippenko 2013) show evidence for late-time ( $>500 \mathrm{~d}$ after maximum brightness) mid-IR emission from warm $\left(T_{\mathrm{d}} \sim 500-800 \mathrm{~K}\right)$ dust. Assuming a simple dust population of a single size $(a=0.1 \mu \mathrm{m})$ graphitic grains yield dust masses of $0.006-0.022 \mathrm{M}_{\odot}$. The dust parameters are most consistent with a pre-existing dust shell that lies beyond the forwardshock radius, most likely radiatively heated by optical and X-ray emission continuously generated by late-time CSM interaction.

\section{SUMMARY AND CONCLUSIONS}

We have analysed the mid-IR light curves of SN 2014J and several other SNe Ia observed with Spitzer, spanning the range from before peak brightness well into the nebular phase. We have characterized, for the first time, the SN Ia light-curve evolution at 3.6 and $4.5 \mu \mathrm{m}$. Our observations indicate that $\mathrm{SNe}$ Ia form a very homogeneous class of objects at these wavelengths, possibly without any lightcurve shape variations. In particular, the mid-IR light curves do not show any evidence for a secondary maximum, as opposed to the case in the near-IR. The latter was investigated by Kasen (2006) to explore the physics of the exploding system. Extending these studies to the now probed longer wavelengths should provide critical tests for SN Ia models.

The Spitzer observations provide a completely new way to test models for the CS environment of SNe Ia and may help in understanding the non-standard reddening measured both for individual SNe Ia and in the large SN Ia samples used to derive cosmological parameters.

By comparing the measured mid-IR fluxes at different epochs for the reddened SNe 2014J, 2006X and 2007le to unreddened SNe, we can place strong constraints on the emission from heated dust within $\sim 10^{18} \mathrm{~cm}$ from the exploding stars. This allows us to exclude the possibility that the bulk of the observed extinction towards these highly reddened SNe Ia is due to CS dust. Foley et al. (2014) claim that half of the extinction $\left(A_{V} \sim 1 \mathrm{mag}\right)$ towards SN 2014J can be attributed to CS dust, while the other half is due to IS dust in $\mathrm{M} 82$. However, our limits on dust emission imply that at most $\tau(V) \approx A_{V} \lesssim 0.1 \mathrm{mag}$ of extinction can be accounted for by CS dust. We emphasize that the derived limits are relatively insensitive to the assumed dust models (as illustrated in Fig. 3). Thus, our findings reaffirm the conclusions from polarization studies of SNe 2014J and 2006X, which indicate that the dust in the line of sight towards these objects is most likely of IS nature (Kawabata et al. 2014; Patat et al. 2015). Furthermore, the lack of heated material in the CS environment of SN 2014J is compatible with the non-detection in X-rays and radio (Margutti et al. 2014; Pérez-Torres et al. 2014). The only comparable previous study of searches for emission from heated CS dust to date was carried out by Johansson, Amanullah \& Goobar (2013), presenting far-IR non-detections of both SNe $2011 \mathrm{fe}$ and $2012 \mathrm{cg}$ using the Herschel Photodetector Array Camera and Spectrometer $70 \mu \mathrm{m}$ instrument. These non-detections exclude CS dust masses $M_{\mathrm{d}} \gtrsim 7 \times 10^{-3} \mathrm{M}_{\odot}$ for dust temperatures $T_{\mathrm{d}} \sim 500 \mathrm{~K}$ at a $3 \sigma$ level for SN $2011 \mathrm{fe}$, while the upper limits are one order of magnitude weaker for SN $2012 \mathrm{cg}$, excluding dust masses $M_{\mathrm{d}} \gtrsim 10^{-1} \mathrm{M}_{\odot}$. Thus, our Spitzer study is more than two orders of magnitude more sensitive than previous attempts.

The mid-IR non-detections for SNe 2006X, 2007le, 2011fe and $2012 \mathrm{cg}$ at late epochs ( $>600 \mathrm{~d}$ after max) can constrain the possible presence of dust at very large radii $\left(>10^{18} \mathrm{~cm}\right)$. These limits could be of value to models proposed by Soker (2014), which seek to explain the time-variable $\mathrm{Na}$ I D absorption seen in SNe 2006X and 2007le, provide limits on the allowed distance to the reflecting dust responsible for light echoes (Wang et al. 2008b; Crotts 2015; Drozdov et al. 2015) or constrain the possibility of newly formed dust grains in SN Ia ejecta (Nozawa et al. 2011).

To summarize, this work expands on previous efforts to study the wide wavelength range of the SED of SNe Ia and provides the first statistical sample of SNe Ia in the mid-IR and a detailed study of the highly reddened object SN 2014J and its CS environment. The non-detection of thermal emission from heated dust in the CS environment of SN 2014J, as well as for SNe 2006X and 2007le where the detection of CS matter at $10^{17} \mathrm{~cm}$ has been claimed, argues against the proposed explanation for the non-standard reddening of SNe Ia invoking multiple scattering on CS dust (Wang 2005; Goobar 2008). Thus, the non-standard reddening may be unrelated to the SN site and originates from the host galaxy ISM being different than what has been derived for the Milky Way, as indicated by Phillips et al. (2013). This could have a serious impact for our understanding of the properties of dust grains in distant galaxies, with profound implications for essentially all areas of extragalactic astronomy.

\section{ACKNOWLEDGEMENTS}

We are grateful to Claes Fransson for stimulating discussions. RA and AG acknowledge support from the Swedish Research Council and the Swedish Space Board. MMK acknowledges support from the Hubble Fellowship and Carnegie-Princeton Fellowship. We acknowledge the John von Neumann Institute for Computing for granting computing time on the supercomputer JUQUEEN at the Jülich Supercomputing Centre. Observations are made with the Spitzer telescope, the Nordic Optical Telescope, operated by the Nordic Optical Telescope Scientific Association at the Observatorio del Roque de los Muchachos, La Palma, Spain, of the Instituto de Astrofisica de Canarias and the Mount Abu 1.2-m Infrared Telescope, India.

\section{REFERENCES}

Amanullah R., Goobar A., 2011, ApJ, 735, 20

Amanullah R. et al., 2014, ApJ, 788, L21

Amanullah R. et al., 2015, MNRAS, 453, 3300 
Ashall C., Mazzali P., Bersier D., Hachinger S., Phillips M., Percival S., James P., Maguire K., 2014, MNRAS, 445, 4427

Betoule M. et al., 2014, A\&A, 568, A22

Blondin S., Prieto J. L., Patat F., Challis P., Hicken M., Kirshner R. P., Matheson T., Modjaz M., 2009, ApJ, 693, 207

Branch D., Doggett J. B., Nomoto K., Thielemann F.-K., 1985, ApJ, 294, 619

Brown P. J. et al., 2015, ApJ, 805, 74

Burns C. R. et al., 2014, ApJ, 789, 32

Cortés J. R., Kenney J. D. P., Hardy E., 2008, ApJ, 683, 78

Crotts A. P. S., 2015, ApJ, 804, L37

Diehl R. et al., 2014, Science, 345, 1162

Dilday B. et al., 2012, Science, 337, 942

Draine B. T., Lee H. M., 1984, ApJ, 285, 89

Drozdov D., Leising M. D., Milne P. A., Pearcy J., Riess A. G., Macri L. M., Bryngelson G. L., Garnavich P. M., 2015, ApJ, 805, 71

Dwek E., 1985, ApJ, 297, 719

Folatelli G. et al., 2010, AJ, 139, 120

Foley R. J. et al., 2012, ApJ, 744, 38

Foley R. J. et al., 2014, MNRAS, 443, 2887

Fox O. D., Filippenko A. V., 2013, ApJ, 772, L6

Fox O. D., Chevalier R. A., Dwek E., Skrutskie M. F., Sugerman B. E. K., Leisenring J. M., 2010, ApJ, 725, 1768

Fox O. D. et al., 2011, ApJ, 741, 7

Friesen B., Baron E., Wisniewski J. P., Parrent J. T., Thomas R. C., Miller T. R., Marion G. H., 2014, ApJ, 792, 120

Gall E. E. E., Taubenberger S., Kromer M., Sim S. A., Benetti S., Blanc G., Elias-Rosa N., Hillebrandt W., 2012, MNRAS, 427, 994

Gerardy C. L. et al., 2007, ApJ, 661, 995

Goobar A., 2008, ApJ, 686, L103

Goobar A., Leibundgut B., 2011, Ann. Rev. Nucl. Part. Sci., 61, 251

Goobar A. et al., 2014, ApJ, 784, L12

Goobar A. et al., 2015, ApJ, 799, 106

Graham M. L. et al., 2015, ApJ, 801, 136

Hicken M. et al., 2009, ApJ, 700, 331

Hildebrand R. H., 1983, Q. J. R. Astron. Soc., 24, 267

Jerkstrand A., Fransson C., Kozma C., 2011, A\&A, 530, A45

Jerkstrand A., Ergon M., Smartt S. J., Fransson C., Sollerman J., Taubenberger S., Bersten M., Spyromilio J., 2015, A\&A, 573, A12

Johansson J., Amanullah R., Goobar A., 2013, MNRAS, 431, L43

Kasen D., 2006, ApJ, 649, 939

Kasliwal M. M. et al., 2017, preprint (arXiv:1701.01151)

Kawabata K. S. et al., 2014, ApJ, 795, L4

Kromer M., Sim S. A., 2009, MNRAS, 398, 1809

Kruegel E., 2003, in Kruegel E., ed., The Physics of Interstellar Dust. The Institute of Physics, Bristol

Laor A., Draine B. T., 1993, ApJ, 402, 441
McClelland C. M., Garnavich P. M., Milne P. A., Shappee B. J., Pogge R. W., 2013, ApJ, 767, 119

Maeda K., Nozawa T., Nagao T., Motohara K., 2015, MNRAS, 452, 3281

Maeda K. et al., 2016, ApJ, 816, 57

Mager V. A., Madore B. F., Freedman W. L., 2013, ApJ, 777, 79

Margutti R., Parrent J., Kamble A., Soderberg A. M., Foley R. J., Milisavljevic D., Drout M. R., Kirshner R., 2014, ApJ, 790, 52

Marion G. H. et al., 2013, ApJ, 777, 40

Marion G. H. et al., 2015, ApJ, 798, 39

Matheson T. et al., 2012, ApJ, 754, 19

Mazzali P. A. et al., 2014, MNRAS, 439, 1959

Milne P. A. et al., 2010, ApJ, 721, 1627

Munari U., Henden A., Belligoli R., Castellani F., Cherini G., Righetti G. L., Vagnozzi A., 2013, New Astron., 20, 30

Nobili S., Goobar A., 2008, A\&A, 487, 19

Nomoto K., Thielemann F.-K., Yokoi K., 1984, ApJ, 286, 644

Nozawa T., Maeda K., Kozasa T., Tanaka M., Nomoto K., Umeda H., 2011, ApJ, 736, 45

Patat F. et al., 2007, Science, 317, 924

Patat F. et al., 2013, A\&A, 549, A62

Patat F. et al., 2015, A\&A, 577, A53

Pereira R. et al., 2013, A\&A, 554, A27

Pérez-Torres M. A. et al., 2014, ApJ, 792, 38

Phillips M. M. et al., 2013, ApJ, 779, 38

Riess A. G. et al., 2011, ApJ, 730, 119

Ritchey A. M., Welty D. E., Dahlstrom J. A., York D. G., 2015, ApJ, 799, 197

Schweizer F. et al., 2008, AJ, 136, 1482

Seitenzahl I. R. et al., 2013, MNRAS, 429, 1156

Silverman J. M. et al., 2012, ApJ, 756, L7

Simon J. D. et al., 2007, ApJ, 671, L25

Simon J. D. et al., 2009, ApJ, 702, 1157

Soker N., 2014, MNRAS, 444, L73

Sternberg A. et al., 2011, Science, 333, 856

Taddia F. et al., 2012, A\&A, 545, L7

Telesco C. M. et al., 2015, ApJ, 798, 93

Wang L., 2005, ApJ, 635, L33

Wang X. et al., 2008a, ApJ, 675, 626

Wang X., Li W., Filippenko A. V., Foley R. J., Smith N., Wang L., 2008b, ApJ, 677, 1060

Weingartner J. C., Draine B. T., 2001, ApJ, 548, 296

Welty D. E., Ritchey A. M., Dahlstrom J. A., York D. G., 2014, ApJ, 792, 106

This paper has been typeset from a $\mathrm{T}_{\mathrm{E}} \mathrm{X} / \mathrm{L} \mathrm{T} \mathrm{E} \mathrm{X}$ file prepared by the author. 